\title{
Afinidades biogeográficas de los galateoideos (Decapoda: Anomura) del Caribe y Pacífico colombiano
}

\section{Biogeographical affinities of Colombian Caribbean and Pacific galateoids (Decapoda: Anomura)}

\author{
Gabriel R. Navas S, ${ }^{1 *}$ Ph.D, Adriana Bermúdez T,1 Ph.D, Catalina Ángel-Yunda, ${ }^{2}$ Biól.Mar, \\ Néstor Hernando Campos, ${ }^{3}$ Ph.D.
}

\begin{abstract}
${ }^{1}$ Universidad de Cartagena, Programa de Biología, Cartagena, Colombia. ${ }^{2}$ Universidad de Wageningen, Maestria en Ciencias Ambientales, Wageningen, Holanda. ${ }^{3}$ Universidad Nacional de Colombia, Sede Caribe, CECIMAR. Santa Marta. *Correspondencia: grnavas@gmail.com
\end{abstract}

Recibido: Septiembre de 2011; Aceptado: Octubre de 2012.

\section{RESUMEN}

Objetivo. Determinar la distribución y afinidades biogeográficas de los galateoideos de aguas colombianas con base en la literatura y muestras recolectadas durante expediciones realizadas entre 1999 y 2002. Materiales y métodos. Se recolectaron ejemplares en 100 estaciones entre 20 y 550 $\mathrm{m}$ de profundidad en el Caribe y Pacífico colombiano. El material recolectado, junto con la información geográfica existente en literatura para las especies conocidas para Colombia se emplearon para realizar mapas de distribución, los cuales se compararon con el "Mapa de ecorregiones marinas del mundo". Resultados. Se obtuvo la información geográfica para 3247 ejemplares recolectados de 18 especies de los géneros Agononida, Anomoeomunida, Munida, Munidoposis y Pleuroncodes, y se complementó con la obtenida a partir de la literatura para las 40 especies de la superfamilia con presencia conocida en aguas colombianas. Se generaron cinco grupos principales de distribución: especies restringidas al Caribe, al Caribe y Atlántico occidental, Anfiatlánticas, restringidas al Pacífico oriental y Anfiamericanas. Se encontró que el $53 \%$ de las especies recolectadas en el Caribe se presentan también en la provincia Atlántico Norte Cálido-Templado, y para el Océano Pacífico la mayor afinidad se da con la provincia Pacífico Oriental Tropical. Conclusiones. Los galateoideos en aguas colombianas presentan ámbitos geográficos y batimétricos amplios, presentándose simpatría entre algunas especies. Las de aguas someras presentan mayores restricciones que aquellas de distribución en aguas profundas. Los mecanismos de distribución de las especies corresponden con el ciclo de vida de cada una y las corrientes predominantes en las provincias.

Palabras clave: Anomura, biogeografía, fauna, Galatheoidea (Fuentes:CAB,AGROVOC). 


\section{ABSTRACT}

Objective. Determine the distribution and biogeographic affinity of the galateoid species found in Colombian waters. The study is based upon bibliographic data and samples collected during expeditions between 1999 and 2002. Materials and methods. Samples of galateoids were collected from 100 locations, from depths ranging between 20 and $550 \mathrm{~m}$, along the Pacific and Caribbean coasts of Colombia. The collected material and geographic information were used to create distribution maps, which were compared with the Marine Ecoregions of the World classification. Results. A total of 3247 specimens of 18 species of the genera Agononida, Anomoeomunida, Munida, Munidoposis y Pleuroncodes were collected in the Caribbean and Pacific regions, and the information was completed with bibliographic data from 40 species of the superfamily with known presence in Colombian waters. Five main distribution groups of species were established: Caribbean, Caribbean and Western Atlantic, pan-Atlantic, Eastern Pacific, and Pan-American. Comparisons with the Marine Ecoregions of The World Map showed that $53 \%$ of the collected species in the Caribbean are also present in the temperate region of the North Atlantic and the East Tropical Pacific province. The greatest affinity among the Colombian Pacific species was found in the Eastern Tropical region of the Pacific Ocean. Conclusions. Ample geographic and bathymetric ranges of Galateoids are present in Colombian marine environments, showing a sympatric distribution among some species. Shallow water species presented more restrictions than deep water ones. The distribution mechanisms of the species correspond with their life cycle and the predominant currents of each province.

Key words: Anomura, biogeography, fauna, Galatheoidea (Sources: CAB, AGROVOC).

\section{INTRODUCCIÓN}

El inventario de las especies marinas de Colombia es un tópico de investigación de gran urgencia debido al creciente impacto que sobre los ecosistemas están causando el cambio climático, el desarrollo no planificado y el aprovechamiento no sostenible, siendo muy probable que se esté perdiendo un gran número de especies que ni siquiera se han llegado a documentar.

Hasta 1995 el conocimiento de la biodiversidad marina en las plataformas y taludes de Colombia era producto de algunas recolectas realizadas en estaciones en la franja costera por exploraciones oceanográficas extranjeras (1). En los últimos años se alcanzó un avance notable en el conocimiento de la megafauna (organismos con tamaños superiores a $20 \mathrm{~mm}$ ) gracias a las exploraciones de la plataforma y talud superior del Caribe colombiano realizadas desde 1995, entre 60 y 900 $\mathrm{m}$ de profundidad, y para el Pacífico frente a las ecorregiones Pacífico norte y Baudó entre los 20 y $500 \mathrm{~m}$ (1).

En las capturas obtenidas las langostillas galateoideas fueron uno de los grupos más importantes en términos de riqueza y abundancia. Estos anomuros poseen un conjunto de caracteres entre los que se destacan el cuerpo simétrico y el abdomen deprimido (Figura 1) (2). Los galateoideos son excepcionalmente diversos, presentando una amplia distribución geográfica y batimétrica en áreas tropicales y templadas (3). Son componentes importantes de las redes tróficas de las plataformas y taludes, y en ciertas regiones algunas especies son utilizadas a nivel comercial (4).

La riqueza, abundancia y amplio ámbito batimétrico y geográfico de los galateoideos presentes en aguas colombianas los hacen apropiados para avanzar en el conocimiento de las afinidades zoogeográficas de los crustáceos anomuros del país.

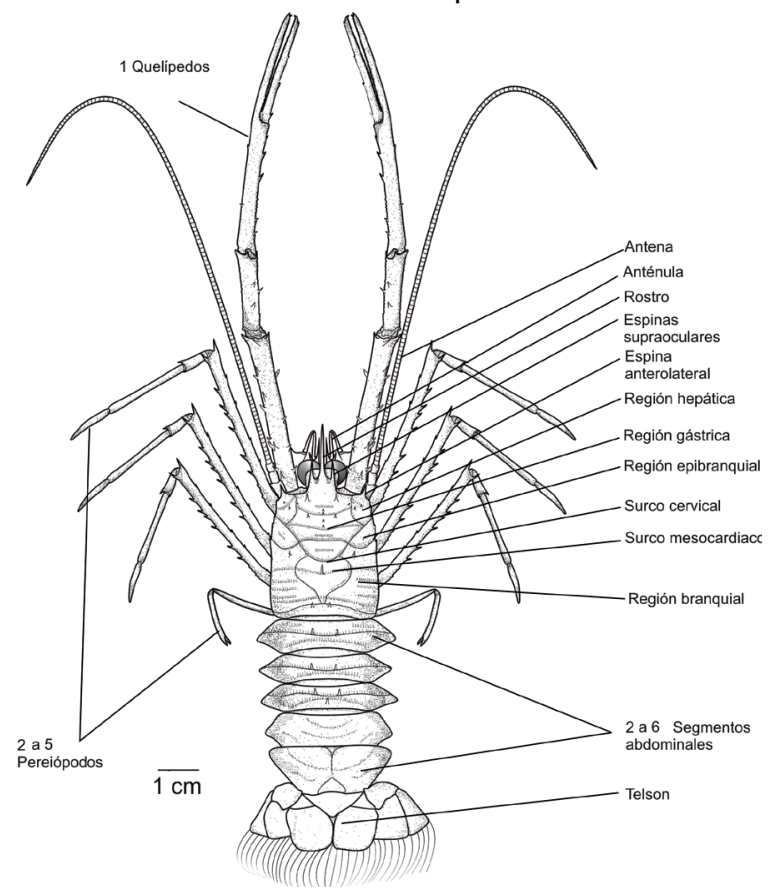

Figura 1. Esquema general de los individuos de la superfamilia Galatheoidea. 


\section{MATERIALES Y MÉTODOS}

Sitio de estudio. Se estudiaron los ejemplares recolectados en 80 estaciones, entre 20 y 550 $\mathrm{m}$ de profundidad, a partir de 160 arrastres realizados entre 1999 y 2002 durante las expediciones Macrofauna I y II a lo largo del Caribe colombiano, a bordo del B/I Ancón; y los recolectados en 40 arrastres en 20 estaciones en 2003 , entre 20 y 500 m durante la expedición Macrofauna III en el norte del Pacífico colombiano a bordo del ARC Malpelo (4). Los organismos fueron recolectados con una red de arrastre demersal tipo "semi-ballon", con dos compuertas metálicas $(91 \mathrm{~cm} \times 63 \mathrm{~cm})$ y red de $9 \mathrm{~m}$ de boca. En cada estación se realizaron dos arrastres de 10 min de duración.

Análisis de ejemplares. Los galateoideos fueron separados por especie, sexados y contados.

Análisis zoogeográfico. Adicional a los especímenes recolectados, se incluyeron los registros publicados de las especies. Cuando existía información sin referencias geográficas puntuales, las coordenadas y/o profundidad fueron tomadas de las bitácoras de los cruceros. A partir de las coordenadas y profundidad de las estaciones donde se capturaron los ejemplares, y de las obtenidas para las especies a partir de las fuentes bibliográficas, se elaboraron mapas de distribución para cada una de las 40 especies conocidas para Colombia empleando las capas disponibles en Google Earth y modelos digitales de profundidad para el Caribe y Pacífico colombiano empleando el programa ETOPO 2. Los mapas de distribución geográfica de las especies fueron comparados gráficamente para determinar grupos con distribuciones congruentes y se elaboró un mapa final general. Cada uno de los grupos fue discutido con base en las provincias y ecorregiones propuestas por Spalding et al (5) en el "Mapa de ecorregiones marinas del mundo" y la sectorización propuesta por Boschi (6) para crustáceos.

\section{RESULTADOS}

Para el Caribe colombiano, en 51 estaciones en un ámbito batimétrico entre 71 y $520 \mathrm{~m}$ de profundidad, se recolectaron 2140 ejemplares de 19 especies de la superfamilia Galatheoidea, agrupadas en cuatro géneros, Anomoeomunida (A. caribensis), Munida ( $M$. constricta, $M$. evermanni, M. flinti, M. forceps, M. irrasa, $M$. pusilla, M. stimpsoni y M. valida), Munidopsis (M. alaminos, M. bradleyi, M. brevimana, M. erinacea, $M$. longimanus, M. platirostris,
M. polita, $M$. ramahtaylorae y $M$. riveroi) y Agononida (A. longipes). Para la parte norte del Pacífico colombiano, en 12 estaciones entre 77 y $474 \mathrm{~m}$ de profundidad, se recolectaron 1107 ejemplares de seis especies, agrupadas en Munida (M. gracilipes, M. mexicana, M. obesa у $M$. refulgens), Munidopsis (M. agassizii) y Pleuroncodes ( $P$. monodon). El género más abundante fue Munida con 12 especies y 1725 ejemplares, seguido por Munidopsis con 10 especies y 403 ejemplares. Agononida longipes fue la más abundante con 1117 individuos, mientras que de Pleuroncodes monodon, cuyos juveniles son planctónicos, solamente se recolectaron dos individuos.

En la figura 2 se presenta la distribución global de las 40 especies de galateoideos con presencia conocida en aguas colombianas, las cuales se distribuyen en regiones tropicales y sub subtropicales ocupando un amplio ámbito batimétrico. Los mapas de distribución de cada una de las especies, fueron previamente descritas por Navas (7).

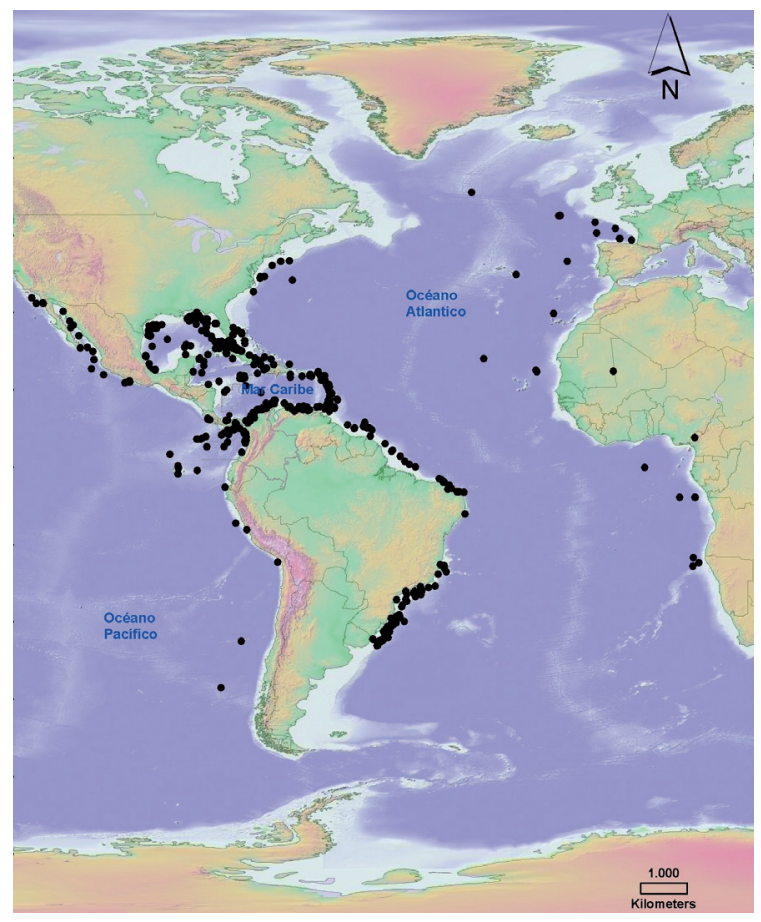

Figura 2. Distribución global de las 42 especies de la superfamilia Galatheoidea conocidas en aguas colombianas.

En la tabla 1 se presenta la distribución de las 40 especies de galateoideos de acuerdo con las Ecorregiones Marinas definidas por Spalding et al (5). En esta clasificación, se delimitaron las siguientes provincias y ecorregiones: Provincia Atlántico Norte Cálido-Templado que incluye las ecorregiones Las Carolinas y Norte del Golfo de México; Provincia Atlántico Tropical Noroccidental, incluye las ecorregiones Bermudas, Bahamas, 
Tabla 1. Distribución de las 40 especies de galateoideos registrados para el Caribe y Pacífico colombianos de acuerdo con las ecorregiones definidas por Spalding et al (5).

\begin{tabular}{|c|c|c|c|}
\hline Especie & \multicolumn{2}{|c|}{ Especies restringidas al Caribe - Ecorregiones } & Ámbito batimétrico $(\mathrm{m})$ \\
\hline Anomoeomunida caribensis & \multicolumn{2}{|l|}{ Antillas Mayores, Caribe Suroccidental. } & $11-38$ \\
\hline Munida evermanni & \multicolumn{2}{|c|}{ Bahamas, Caribe Oriental, Antillas Mayores, Caribe Suroccidental. } & $230-475$ \\
\hline Munidopsis brevimana & \multicolumn{2}{|c|}{$\begin{array}{l}\text { Antillas Mayores, Caribe Suroccidental, Caribe Oriental, Caribe Sur, Antillas Mayores, Golfo } \\
\text { de México. }\end{array}$} & $365-878$ \\
\hline Munidopsis colombiana & \multicolumn{2}{|l|}{ Caribe Sur. } & 4151 \\
\hline Munidopsis ramahtaylorae & \multicolumn{2}{|c|}{ Caribe Sur, Antillas Mayores, Golfo de México, Caribe Occidental. } & $368-834$ \\
\hline Munidopsis reynoldsi & \multicolumn{2}{|l|}{ Caribe Sur, Antillas Mayores. } & 4151 \\
\hline Munidopsis riveroi & \multicolumn{2}{|c|}{ Caribe Sur, Florida, Antillas Mayores, Caribe Occidental. } & $134-732$ \\
\hline Munidopsis spinoculata & \multicolumn{2}{|c|}{ Caribe Sur, Antillas Mayores, Florida, Golfo de México, Caribe Occidental. } & $475-1506$ \\
\hline \multicolumn{4}{|c|}{ Especies con distribución Caribeña y Atlántico Occidental - Ecorregiones } \\
\hline Agononida longipes & \multicolumn{2}{|c|}{$\begin{array}{l}\text { Caribe Oriental, Bahamas, Brasil Nororiental, Brasil Oriental, Caribe Sur, Antillas Mayores, } \\
\text { Florida, Golfo de México, Guyana, Caribe Oriental, Antillas Mayores, Carolina, Golfo de } \\
\text { México. }\end{array}$} & $188-712$ \\
\hline Galacantha spinosa & \multicolumn{2}{|c|}{ Caribe Sur, Caribe Oriental, Florida, Guyana. } & $600-2900$ \\
\hline Munida angulata & \multicolumn{2}{|c|}{ Antillas Mayores, Caribe Sur, Brasil Nororiental, Brasil Oriental, Florida, Golfo de México. } & $24-75$ \\
\hline Munida constricta & \multicolumn{2}{|c|}{ Antillas Mayores, Brasil Nororiental, Brasil Oriental, Caribe Sur, Caribe Oriental. } & $277-835$ \\
\hline Munida flinti & \multicolumn{2}{|c|}{$\begin{array}{l}\text { Antillas Mayores, Brasil Nororiental, Brasil Oriental, Caribe Sur, Caribe Oriental, Golfo de } \\
\text { México, Guyana, plataforma Uruguay-Buenos Aires. }\end{array}$} & $\begin{array}{l}11-630 \\
\text { El registro a } 11 \mathrm{~m} \text { es } \\
\text { dudoso por ser un único } \\
\text { ejemplar }\end{array}$ \\
\hline Munida forceps & \multicolumn{2}{|c|}{$\begin{array}{l}\text { Antillas Mayores, Brasil Nororiental, Brasil Oriental, Caribe Sur, Caribe Oriental, Golfo de } \\
\text { México, Florida, Guyana, Plataforma Uruguay-Buenos Aires. }\end{array}$} & $30-950$ \\
\hline Munida irrasa & \multicolumn{2}{|c|}{$\begin{array}{l}\text { Antillas Mayores, Bermuda, Caribe Oriental, Brasil Nororiental, Brasil Oriental, Caribe Sur, } \\
\text { Florida, Golfo de México, Carolinas, Plataforma Uruguay-Buenos Aires. }\end{array}$} & $15-475$ \\
\hline Munida pusilla & \multicolumn{2}{|c|}{$\begin{array}{l}\text { Antillas Mayores, Caribe Oriental, Brasil Nororiental, Brasil Oriental, Caribe Sur, Florida, } \\
\text { Golfo de México. }\end{array}$} & $33-135$ \\
\hline Munida stimpsoni & \multicolumn{2}{|c|}{$\begin{array}{l}\text { Bahamas, Caribe Oriental, Caribe Sur, Caribe Suroccidental, Antillas Mayores, Florida, } \\
\text { Golfo de México. }\end{array}$} & $110-2000$ \\
\hline Munida valida & \multicolumn{2}{|c|}{$\begin{array}{l}\text { Antillas Mayores, Brasil Nororiental, Brasil Oriental, Caribe Sur, Florida, Golfo de México, } \\
\text { Guayana, Carolinas. }\end{array}$} & $90-2300$ \\
\hline Munidopsis abbreviata & \multicolumn{2}{|c|}{ Antillas Mayores, Caribe Sur, Caribe Suroccidental, Florida, Golfo de México. } & $670-1345$ \\
\hline Munidopsis alaminos & \multicolumn{2}{|c|}{ Caribe Sur, Caribe Suroccidental, Guayana, Golfo de México. } & $423-828$ \\
\hline Munidopsis armata & \multicolumn{2}{|c|}{ Antillas Mayores, Caribe Suroccidental, Caribe Sur, Florida, Golfo de México, Guayana. } & $302-1446$ \\
\hline Munidopsis bradleyi & \multicolumn{2}{|c|}{$\begin{array}{l}\text { Antillas Mayores, Bahamas, Caribe Sur, Caribe Suroccidental, Carolinas, Norte del Golfo } \\
\text { de México. }\end{array}$} & $465-914$ \\
\hline Munidopsis erinacea & \multirow{2}{*}{\multicolumn{2}{|c|}{$\begin{array}{l}\text { Antillas Mayores, Brasil Nororiental, Brasil Suroriental, Caribe Sur, Caribe Suroccident } \\
\text { Florida, Golfo de México. } \\
\text { Antillas Mayores, Caribe Oriental, Caribe Sur, Caribe Suroccidental, Florida, Golfo de } \\
\text { México. }\end{array}$}} & $300-1014$ \\
\hline Munidopsis longimanus & & & $292-1748$ \\
\hline Munidopsis platirostris & $\begin{array}{l}\text { Antillas Mayores, Caribe Oriental, Caribe Sur, Car } \\
\text { México, Carolinas. }\end{array}$ & be Suroccidental, Florida, Golfo de & $182-1280$ \\
\hline Munidopsis polita & $\begin{array}{l}\text { Brasil Nororiental, Brasil Oriental, Caribe Sur, Car } \\
\text { Caribe Occidental, Florida, Golfo de México. }\end{array}$ & be Suroccidental, Caribe Oriental, & $132-977$ \\
\hline Munidopsis sigsbei & $\begin{array}{l}\text { Antillas Mayores, Brasil Oriental, Brasil Nororienta } \\
\text { Florida, Golfo de México, Guayana, Caribe Orienta }\end{array}$ & $\begin{array}{l}\text { I, Caribe Sur, Caribe Suroccidental, } \\
\text { I. }\end{array}$ & $755-1818$ \\
\hline Munidopsis simplex & $\begin{array}{l}\text { Bahamas, Caribe Oriental, Caribe Sur, Caribe Sur } \\
\text { México. }\end{array}$ & ccidental, Antillas Mayores, Golfo de & $608-3970$ \\
\hline Munidopsis subspinoculata & Caribe Sur, Golfo de México, Antillas Mayores. & & $475-823$ \\
\hline & Especies con distribución restringida al & Pacífico Oriental - Localidades & \\
\hline Galacantha diomedeae & $\begin{array}{l}\text { Chile Central, Ensenada de Panamá, Nicoya, Guay } \\
\text { Tropical Mexicano, Ensenada de California del Sur }\end{array}$ & $\begin{array}{l}\text { quil, Galápagos, Cortez, Pacífico } \\
\text { Transición del Magdalena. }\end{array}$ & $1206-1570$ \\
\hline Munida gracilipes & Perú Central, Ensenada de Panamá, Nicoya. & & $115-300$ \\
\hline Munida mexicana & Ensenada de Panamá, Guayaquil, Galápagos, Cor & ez, Pacífico Tropical Mexicano. & $35-150$ \\
\hline Munida obesa & Ensenada de Panamá, Nicoya, Perú Central. & & $150-330$ \\
\hline Munida refulgens & Ensenada de Panamá, Guayaquil, Nicoya, Cortez, & Pacífico Tropical Mexicano. & $95-300$ \\
\hline Munidopsis agassizii & Ensenada de Panamá, Perú Central. & & $210-580$ \\
\hline Munidopsis latirostris & $\begin{array}{l}\text { Pacífico Tropical Mexicano, Ensenada de Panamá, } \\
\text { Transición del Magdalena. }\end{array}$ & Ensenada de California del Sur, Cortez, & $280-3240$ \\
\hline Pleuroncodes monodon & Chile Central, Ensenada de Panamá, Nicoya, Pacíf & ico Tropical Mexicano. & $172-523$ \\
\hline & Especies con distribución en el Atlánti & Occidental y Pacífico Oriental & \\
\hline Especie & Atlántico occidental & Pacífico oriental & Ámbito batimétrico $(\mathrm{m})$ \\
\hline Galacantha rostrata & $\begin{array}{l}\text { Antillas Mayores, Caribe Suroccidental, Florida, } \\
\text { Guyana, Carolinas, Norte del Golfo de México. }\end{array}$ & $\begin{array}{l}\text { Chile Central, Nicoya, Guayaquil, } \\
\text { Galápagos, Cortez, Pacífico Tropical } \\
\text { Mexicano. }\end{array}$ & $250-4800$ \\
\hline Munidopsis aries & Caribe Sur y Oriental. & $\begin{array}{l}\text { Ensenada de California del Sur, Cortez, } \\
\text { Transición del Magdalena. }\end{array}$ & $2900-5320$ \\
\hline Munidopsis subsquamosa & $\begin{array}{l}\text { Caribe Suroccidental, Golfo de México, Antillas } \\
\text { Mayores. }\end{array}$ & $\begin{array}{l}\text { Nicoya, Galápagos, Pacífico Tropical } \\
\text { Mexicano, Ensenada de Panamá. }\end{array}$ & $2513-3428$ \\
\hline
\end{tabular}


Caribe Oriental, Antillas Mayores, Caribe Sur, Caribe Suroccidental, Caribe Occidental, Sur del Golfo de México y Florida; Provincia Plataforma Norte de Brasil, incluye las ecorregiones Guayana y Amazonia; Provincia Atlántico Tropical Suroccidental, incluye las ecorregiones Islas Sao Pedro y Sao Paulo, Fernando de Naronha y Atolón Las Rocas, Brasil Nororiental, Brasil Oriental e Islas Trinidad y Martin Vaz; Provincia Atlántico Suroccidental Cálido-Templado que incluye las ecorregiones Brasil Suroriental, Río Grande, Río de la Plata, Plataforma Uruguay Buenos Aires; Provincia Pacífico Tropical Oriental que incluye las ecorregiones Revillagigedo, Clipperton, Pacífico Tropical Mexicano, ChiapasNicaragua, Nicoya, Isla de Cocos, Ensenada de Panamá, Guayaquil; Provincia Galápagos con sus respectivas ecorregiones Norte, Oriente y Occidente; Provincia del Pacífico Nororiental Cálido-Templado, incluye las ecorregiones Ensenada de California del Sur, Cortez y Transición del Magdalena; Provincia Pacífico Suroriental Cálido-Templado con las ecorregiones Perú Central, Humboldt, Chile Central y Araucaria; Provincia Juan Fernández y Desventuradas con la ecorregión del mismo nombre.

Especies con distribución Caribe. Restringidas al Caribe, una especie pertenece al género Munida, seis a Munidopsis y una al género monoespecífico Anomoeomunida. A. caribensis es la especie del grupo que alcanza aguas más someras ( 11 $\mathrm{m})$, mientras que Munidopsis colombiana y $M$. reynoldsi alcanzan profundidades mayores a $4000 \mathrm{~m}$ (Tabla 1).

Especies con distribución Caribe y Atlántico occidental. Pertenecen a los géneros Munidopsis con 11 especies, Munida con ocho, y Agononida y Galacantha con una cada uno. Cinco especies del género Munida fueron recolectadas a profundidades menores a $100 \mathrm{~m}$, de las cuales Munida angulata $(24 \mathrm{~m})$, M. pusilla $(33 \mathrm{~m})$ y M. irrasa $(15 \mathrm{~m})$ han sido registradas en aguas más someras. Ocho especies del género Munidopsis se distribuyen a más de $1000 \mathrm{~m}$ de profundidad y en el caso de $M$. simplex los individuos han sido recolectados cerca de $4000 \mathrm{~m}$ (Tabla 1).

Especies con distribución anfiatlántica. Munidopsis crassa se distribuye en el Atlántico Occidental desde Carolina del Norte, Bahamas, la península de Yucatán, el Caribe, Santa Cruz, Colombia y Venezuela hasta el Atlántico Oriental en Suráfrica, en la bahía de Biscaya, Azores y las Islas Canarias. Su ámbito batimétrico es muy amplio, entre 2524 y 5315 m de profundidad (7). Munidopsis geyeri se distribuye en el Atlántico Oriental, en Angola y en el Atlántico Occidental en el golfo de México y el Caribe en Jamaica, Haití,
Colombia y Venezuela, entre 2600 y 4151 m de profundidad (7).

Especies con distribución Pacífico oriental. Para el Pacífico oriental colombiano se conocen cuatro especies del género Munida, dos de Munidopsis, una de Galacantha y una de Pleuroncodes. Munida mexicana y M. refulgens se registraron a 35 y 95 $\mathrm{m}$, considerándose especies someras. La especie de aguas profundas Galacantha diomedeae ha sido recolectada a $1570 \mathrm{~m}$ y Munidopsis latirostris a $3240 \mathrm{~m}$ (Tabla 1).

Especies con distribución Anfiamericana. Se han registrado dos especies del género Munidopsis y una de Galacantha, todas ellas de aguas profundas. Munidopsis aries es la que ha sido registrada a mayor profundidad, a $5320 \mathrm{~m}$ (Tabla 1).

Distribución de acuerdo con el sistema de ecorregiones marinas del mundo. Según la propuesta de Spalding et al (5) en el sistema "Marine Ecoregions Of the World" (MEOW, por sus siglas en Inglés) (Figura 3 a y b), la costa Caribe colombiana está incluida en el dominio Atlántico tropical en la provincia Atlántico tropical noroccidental; la península de la Guajira hace parte de la ecorregión Caribe sur y la parte al sur de la Guajira está incluida en la ecorregión Caribe suroccidental. Solo Munidopsis colombiana se encontró restringida a la ecorregión Caribe suroccidental, del resto, el $53 \%$ de las especies se presentaron también en la provincia Atlántico norte cálido-templado y el $47 \%$ en la Plataforma Norte de Brasil (PNB). La provincia de menor afinidad con el Caribe colombiano fue Atlántico suroccidental cálido templado con el $18 \%$ (Figura 4).

Provincia Atlántico tropical noroccidental. Anomoeomunida caribensis, Munida evermanni, Munidopsis brevimana, M. colombiana, $M$. ramahtaylorae, $M$. reynoldsi, $M$. riveroi y $M$. spinoculata están restringidas a esta provincia. Sus aguas cálidas son influenciadas por la corriente oceánica del golfo y su ubicación tropical ocasiona que el agua superficial se mantenga entre 21 y $32^{\circ} \mathrm{C}$ durante el año.

Provincia Atlántico norte cálido-templado (c). Incluye el $53 \%$ de las especies registradas para el Caribe colombiano, Agononida longipes, Galacantha rostrata, Munida angulata, M. flinti, M. forceps, M. irrasa, M. pusilla, M. stimpsoni, M. valida, Munidopsis alaminos, M. crassa, $M$. erinacea, M. platirostris, M. polita, M. sigsbei, M. simplex y $M$. subsquamosa se distribuyen también en la provincia Atlántico norte cálido-templado, encontrándose en las ecorregiones norte del golfo de México y Las Carolinas. 

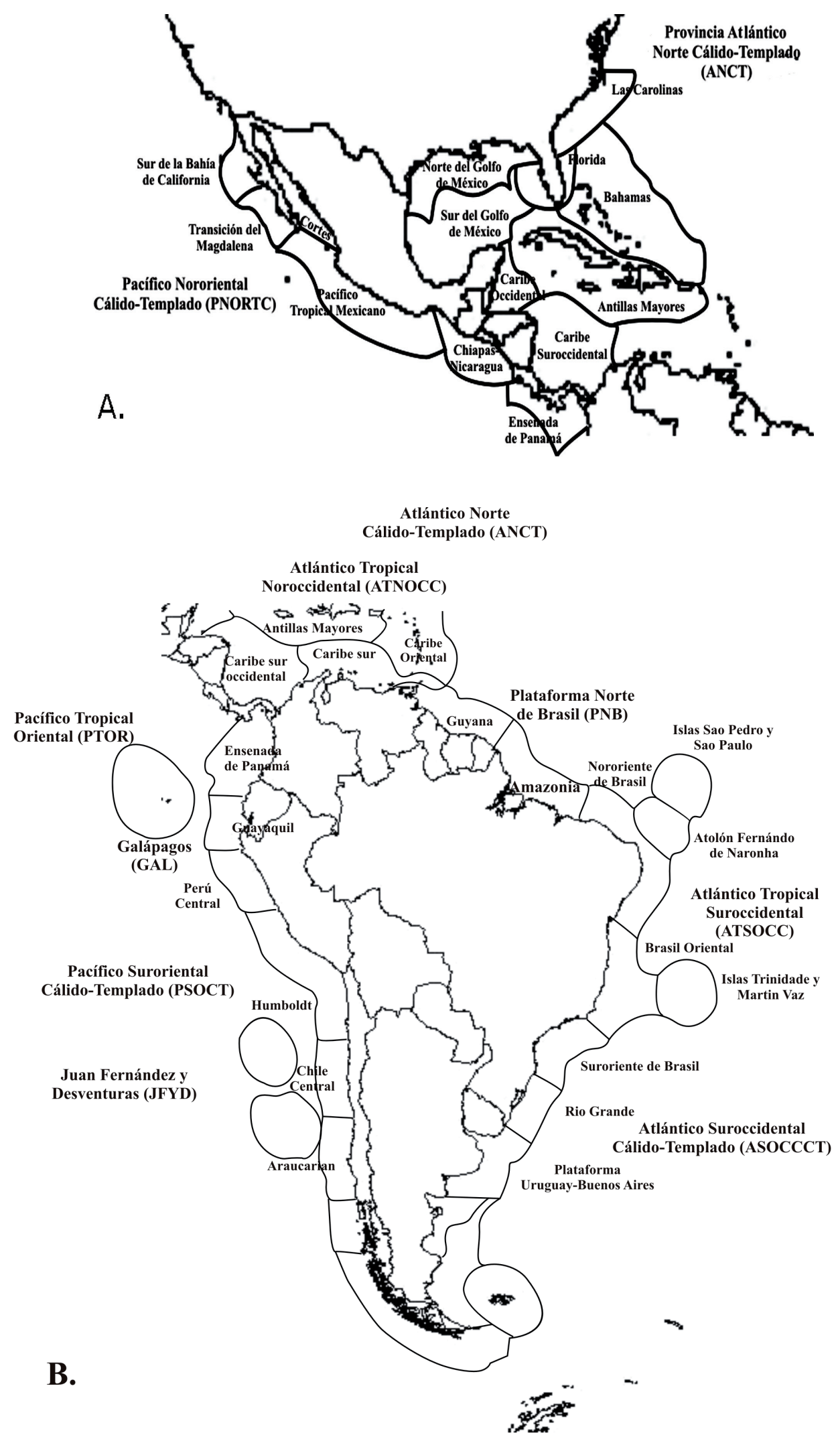

Figura 3. Esquema modificado de los dominios, provincias y ecorregiones del sistema "Ecorregiones marinas del mundo" (MEOW, por sus siglas en Inglés) establecido por Spalding et al (5). A. América del Norte; B. Sur América. 


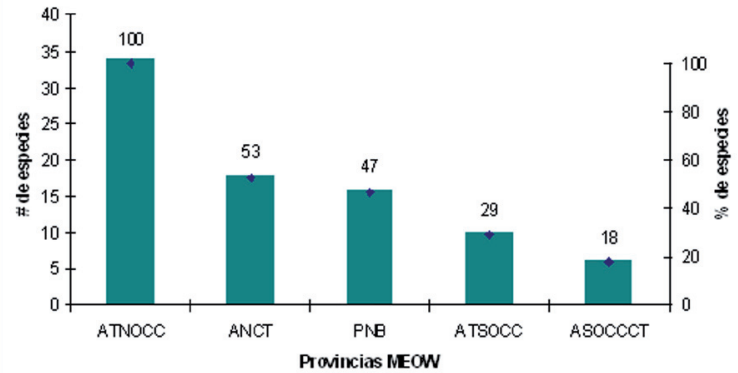

Figura 4. Número y porcentaje de especies de la superfamilia Galatheoidea registradas para el Caribe colombiano con relacion al total de especies presentes en cada una de las provincias marinas establecidas por Spalding et al (5) en el sistema de sectorización de ecorregiones marinas del mundo, Marine Ecoregions of the World (MEOW). Las abreviaturas corresponden a las siguientes Provincias: ATNOCC: Atlántico Tropical Noroccidental; ANCT: Atlántico Norte Cálido-Templado; PNB: Plataforma Norte de Brasil; ATSOCC: Atlántico Tropical Suroccidental; ASOCCCT: Atlántico Suroccidental Cálido-Templado.

La confluencia de estas dos ecorregiones le confiere a la región características climáticas particulares, además de estar sometida a la influencia simultánea de corrientes frías y tropicales. La ecorregión norte del golfo de México presenta condiciones climáticas y oceanográficas diferentes a las del resto de la región tropical. Se presentan dos épocas climáticas marcadas en el año, en otoño-invierno (noviembre-marzo) los vientos provenientes del norte, generados por masas polares, disminuyen la temperatura superficial del agua hasta $11^{\circ} \mathrm{C}$ en la parte norte del golfo, mientras que en el sur durante la misma época, se registran temperaturas hasta de $21^{\circ} \mathrm{C}$. En verano la temperatura se eleva hasta los $30^{\circ} \mathrm{C}$ de manera homogénea en el golfo (6).

La ecorregión Las Carolinas se considera un área de aguas cálido-templadas influenciada por la corriente cálida del golfo que fluye en sentido norte y la corriente fría de Labrador que corre en sentido sur, creando constantemente una mezcla de aguas. Este fenómeno también está sujeto a los cambios climáticos de las épocas de invierno y verano, presentándose temperaturas entre 20 y $25^{\circ} \mathrm{C}$ y entre 28 y $30^{\circ} \mathrm{C}$ respectivamente (6).

Provincia plataforma norte de Brasil. El $47 \%$ de las especies registradas para el Caribe colombiano se encuentran también distribuidas en las ecorregiones Guyana y Amazonía de la provincia plataforma norte de Brasil; éstas son: Agononida longipes, Galacantha spinosa, Munida

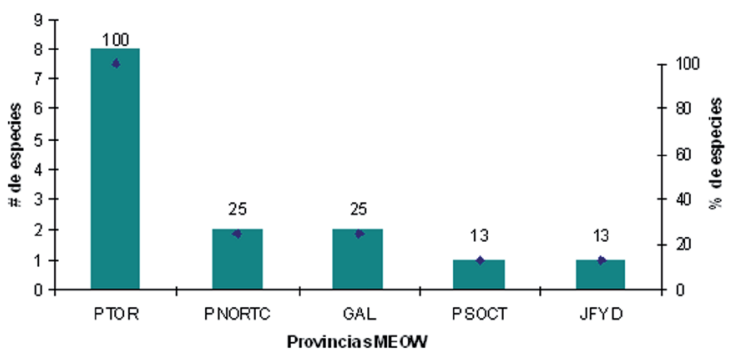

Figura 5. Número y porcentaje de especies de la superfamilia Galatheoidea registradas para el Pacífico colombiano con relación al total de especies presentes en cada una de las provincias marinas establecidas por Spalding et al (5) en el sistema de sectorización de ecorregiones marinas del mundo, Marine Ecoregions of the World (MEOW). Provincias: PNORCT: Pacífico Nororiental Cálido-Templado; PTOR: Pacífico Tropical Oriental; GAL: Galápagos; PSOCT: Pacífico Suroriental Cálido-Templado; JFYD: Juan Fernández y Desventuradas.

constricta, M. forceps, M. irrasa, M. pusilla, M. valida, Munidopsis abbreviata, $M$. alaminos, $M$. armata, M. erinacea, M. geyeri, $M$. longimanus, $M$. platirostris, M. sigsbei y M. simplex. Considerada una provincia de transición, el inicio de la ecorregión Guyana a los $10^{\circ}$ de latitud norte marca la división entre Atlántico norte y Atlántico sur, representando el Ecuador biogeográfico. Se presenta una importante descarga de ríos como el Orinoco, Amazonas, Pará, Tocantins y Parnaíba que reducen la producción primaria y transportan grandes cantidades de materia orgánica. De igual manera, en ambas ecorregiones se presenta una reducción de salinidad y la alteración del tipo de fondo por sedimentos continentales. Estas condiciones generan una barrera oligosalina que restringe el paso de las especies en la mayoría de los casos (8).

Provincia Atlántico tropical suroccidental. En esta se presenta el $29 \%$ de las especies registradas para el Caribe colombiano: Munida angulata, M. constricta, M. flinti, M. forceps, $M$. irrasa, M. pusilla, M. valida, Munidopsis erinacea, M. polita y M. sigsbei. Conformada por cinco ecorregiones del sistema MEOW, las aguas de esta provincia son modificadas principalmente al norte por la corriente de las Guyanas que fluye hacia el Caribe y al sur la corriente de Brasil con masas de agua de $25^{\circ} \mathrm{C}$ y salinidad de 36.5. Bajo la corriente de Brasil, cerca de los $200 \mathrm{~m}$ de profundidad, corre una contracorriente de origen subtropical con temperaturas y salinidades más bajas (8). 
Provincia Atlántico suroccidental cálidotemplado. Es la provincia con menor afinidad con el $18 \%$ de las especies registradas para Colombia. Se encuentran Agononida longipes, M. constricta, M. flinti, M. forceps, M. irrasa y $M$. valida. Incluye las aguas costeras de la Patagonia, el litoral norte de Buenos Aires y Uruguay hasta $23^{\circ} \mathrm{C}$ en el sur de Brasil. Toda la región está influenciada por la interacción entre la corriente Malvinas que fluye sobre el talud desde el sur, con aguas subantárticas ricas en nutrientes y la corriente de Brasil proveniente del norte con masas de agua de $20^{\circ} \mathrm{C}$ y salinidades de 36 , y que se desplaza por fuera de la plataforma (6).

Costa Pacífica colombiana. Hace parte de la ecorregión ensenada panámica de la provincia Pacífico oriental tropical. De las ocho especies de la superfamilia registradas para el Pacífico colombiano, solamente Galacantha diomedeae y Munida mexicana se distribuyen también en las provincias Pacífico nororiental cálido-templado y Galápagos. Las provincias Juan Fernández y Desventuradas y Pacífico suroriental cálidotemplado comparten a G. diomedeae siendo ésta la única que se presenta en las cinco provincias del Pacífico oriental, y que extiende su ámbito geográfico hasta el sur del continente Americano (Figura 5).

Provincia Pacífico tropical oriental. Es la zona con la mayor riqueza de especies del Pacífico tropical. La temperatura oscila entre 19 y $28^{\circ} \mathrm{C}$ en general, en invierno puede caer a $14^{\circ} \mathrm{C}$ en la parte norte y en verano elevarse hasta los 30 o $32^{\circ} \mathrm{C}(6)$.

Provincia Pacífico nororiental cálidotemplado y provincia Galápagos. La primera provincia se encuentra al norte con las ecorregiones sur de la bahía de California, Cortés y transición del Magdalena, en la costa occidental del continente americano. Se caracterizan por temperaturas entre los 13 y $25^{\circ} \mathrm{C}$ que corresponden a aguas cálido-templadas. La corriente de California transporta aguas frías a lo largo de la costa de California hasta Concepción en donde la temperatura disminuye marcadamente y la corriente desaparece fuera de la costa. Esto determina un agudo gradiente de temperatura que se incrementa hacia el sur y cambia la composición faunística formando una zona de transición (6).

De igual manera, en la parte oceánica noroccidental de Suramérica, la provincia Galápagos presenta a G. diomedeae y M. mexicana. Este archipiélago, situado a aproximadamente $1000 \mathrm{~km}$ de la costa del Ecuador, presenta una temperatura superficial promedio mensual de $23.2^{\circ} \mathrm{C}$ y en mayo de $24.5^{\circ} \mathrm{C}$, en el mes de marzo alcanza niveles muy altos hasta de $25.5^{\circ} \mathrm{C}$. En los años en los que se presenta el fenómeno El Niño llega hasta $29.0^{\circ} \mathrm{C}(6)$.

Provincia Pacífico suroriental cálidotemplado y Provincia Juan Fernández y Desventuradas. Estas se ubican en la zona templada del occidente suramericano abarcando la costa de Perú y Chile, encontrándose solamente Galacantha diomedeae, por lo cual se pueden considerar las provincias menos afines con el Pacífico colombiano. La especie ha sido recolectada frente a Valparaíso, localidad influenciada por la corriente de Humboldt que transporta aguas ricas en nutrientes desde la isla Chiloé en dirección norte hacia Perú con temperaturas entre los 11 y $19^{\circ} \mathrm{C}$ (6).

\section{DISCUSIÓN}

Los resultados obtenidos demuestran la amplia distribución batimétrica y las tendencias generales de los géneros y la mayoría de especies a ocupar la zona mesopelágica, un patrón conocido en otras localidades (3). Las especies se reemplazan en el gradiente batimétrico de manera solapada, como se observa también en el golfo de México (9). En el Caribe colombiano Anomoeomunida caribensis es la especie más somera, seguida por especies de los géneros Munida, Agononida, Munidopsis y Galacantha, siendo estas últimas las que alcanzan mayor profundidad. Los géneros más ricos en especies como Munidopsis y Munida presentan también ámbitos batimétricos más amplios; sin embargo, grupos de especies similares morfológicamente y que comparten los mismos hábitats (sintópicas), tienen ámbitos batimétricos más restringidos, como en el caso de Munida flinti, M. stimpsoni y $M$. evermanni o Munidopsis brevimanus y $M$. longimanus.

En general se observó que especies que habitan aguas superficiales tienen distribuciones más restringidas que las de aguas más profundas (3,10-12); en los extremos se puede citar a Anomoeomunida caribensis, restringida al sur del Caribe a $11 \mathrm{~m}$, y a Munidopsis subsquamosa y M. aries que alcanzan $3427 \mathrm{~m}$ y $5320 \mathrm{~m}$ respectivamente, distribuidas en el Pacífico oriental desde la costa occidental de los Estados Unidos hasta el sur en Chile, en el Pacífico occidental hasta Japón y en varias localidades del Atlántico oriental.

No se encontró evidencia de que la pluma de descarga del río Magdalena actúe como una 
barrera zoogeográfica para las especies de galateoideos presentes en el Caribe colombiano.

La amplia distribución de las especies de aguas profundas se puede deber, al menos en parte, a la homogeneidad de los fondos abisales, la no existencia de barreras absolutas para la dispersión, la baja influencia de los cambios climáticos y oceanográficos (13), y al movimiento lento y gradual de las masas continentales (14), lo cual aunado a una baja tasa de mutación genética, favorecería en menor medida los procesos de especiación. Se ha postulado que la especiación en organismos de aguas profundas se puede atribuir principalmente a la distancia (14).

El patrón de distribución de los galateoideos incluidos en este estudio refleja las características principales de su ciclo de vida. En su mayoría son especies bentónicas, móviles, con un espectro alimenticio amplio y un desarrollo larval complejo con varios estadios, algunos de los cuales pueden estar ausentes en algunas especies $(15,16)$.

Estas características les permiten a los individuos establecerse y en algunos casos, abundar en los fondos blandos del talud. En las zonas por debajo de los $200 \mathrm{~m}$, debido a la estabilidad de los ambientes y la casi ausencia de barreras absolutas, el factor limitante para la dispersión de las especies podría ser la influencia de las masas de agua presentes en las diferentes regiones y las estrategias reproductivas. El movimiento de dichas masas puede restringir o promover la dispersión de larvas entre provincias o ecorregiones, ocasionando la colonización o recolonización de hábitats, y garantizando el flujo genético entre ellas (17).

Se determinó una mayor riqueza en el Caribe que en el Pacífico oriental tropical. Se ha postulado que el Caribe ha experimentado una historia geológica compleja que ha generado una plataforma continental mucho más amplia y variable, lo que probablemente genera ambientes óptimos a distintas profundidades para el asentamiento de una mayor cantidad de especies de más géneros. Además, en el Caribe los organismos han tenido varios procesos de colonización y extinción por causa del enfriamiento del océano, en las glaciaciones. Además, desde el cierre del pasaje marino en Panamá, la región experimentó cambios intensos de temperatura en sus aguas y en el nivel del mar. La menor cantidad de especies y géneros en el Pacífico se puede atribuir a la plataforma continental estrecha, con ambientes más homogéneos y menor mezcla de masas de agua (18).
Atlántico. Al menos para las especies del talud superior del género Munida la provincia Atlántico Tropical Noroccidental puede constituirse en una zona de especiación debido a la gran variedad de ecosistemas que confluyen en ella (19).

Asimismo, se puede observar una restricción en la distribución de varias de ellas, pues las ocho presentes en aguas colombianas parecen no dispersarse hacia las aguas cálido-templadas del sur o del norte (8). Una de las corrientes que influye en esta Provincia, y que además podría estar limitando la distribución de algunas de las especies en la región del Caribe, es la corriente de las Guyanas. Esta fluye paralela a la plataforma brasilera en dirección sur-norte hacia el Caribe, arrastrando aguas frías hasta las Antillas. Un ramal se desprende de ella y sigue por el lado externo de las Antillas, hasta la península de Yucatán, que posteriormente hace un giro anticiclónico local y se convierte en la corriente de la Florida. Esta última, podría ser la responsable de la delimitación septentrional de la distribución de las especies (8).

Agononida longipes, Munida flinti y M. valida también alcanzan a tolerar las aguas cálidotempladas de la provincia Atlántico norte cálidotemplado. La presencia de estas especies en la ecorregión Golfo de México se puede justificar en términos del transporte de organismos durante las fases larvales a través de las aguas tropicales de la corriente del Caribe o por la corriente de la Florida. La corriente del Caribe logra entrar al golfo de México por la parte sur-oriental, alcanzando el extremo norte, mientras que la corriente de la Florida pasa entre el sur de la península y el norte de Cuba hacia el nororiente hasta la ecorregión Las Carolinas, bañando las costas de Carolina del Norte y Virginia $(6,8)$. A pesar de la temperatura más baja en estas zonas, al parecer estas especies tienen un espectro amplio de tolerancia, encontrándose en la misma región a diferentes profundidades con intervalos desde los $20^{\circ} \mathrm{C}$ entre 100 y 150 $\mathrm{m}$ de profundidad (preferida por $M$. flinti) hasta $12^{\circ} \mathrm{C}$ cerca de la franja de los $300 \mathrm{~m}$ (preferida por $A$. longipes y $M$. valida), lo que sugiere que la temperatura no es un factor limitante en la dispersión de sus larvas.

El desplazamiento de las especies de las provincias ubicadas en aguas tropicales hacia otras regiones del sur, ocurre en dirección contraria al de las principales corrientes superficiales, estando este movimiento relacionado a las respectivas contracorrientes. En la provincia Plataforma Norte de Brasil, hacia el sur, en aguas someras la desembocadura de los ríos de la ecorregión Guyanas, podrían constituirse en barreras a 
la dispersión de las especies, sin embargo, tratándose de especies de aguas profundas del talud superior continental, la mencionada influencia es menor que en la plataforma. Así Munida angulata, $M$. constricta, M. flinti, $M$. forceps, M. irrasa, M. pusilla, M. valida, Munidopsis reynoldsi, $M$. polita y $M$. sigsbei pueden superar estas barreras y ampliar su distribución hacia el sur hasta la Provincia Atlántico tropical suroccidental (8). Las condiciones menos favorables para la dispersión de los galateoideos presentes en la Provincia Atlántico suroccidental cálido-templado, restringe el número de especies a solo cinco, la mayoría representantes del género Munida (6).

Pacífico. Las especies más someras aparentemente, están más relacionadas con la provincia Pacífico oriental tropical del océano Pacífico y no parece observarse una dispersión hacia el Pacífico occidental. Es posible que las especies presentes hayan sido transportadas a las zonas, donde actualmente se encuentran, en la medida en que se movieron los fondos oceánicos a través del movimiento de las placas tectónicas, o provienen del Caribe a través del istmo de Panamá.

De las ocho especies presentes en la provincia Pacífico tropical oriental, Galacantha diomedeae y Munida mexicana presentan una distribución desde el golfo de California con numerosos registros al norte, mientras que con menores cantidades, Galacantha diomedae se dispersa solamente hacia las provincias del sur de América a lugares como Ecuador, Perú y Chile. En esta región del Pacífico occidental, las especies se encuentran sujetas a fuertes variaciones estacionales ocasionadas por la corriente de Humboldt y fenómenos recurrentes como el Niño. Además del sistema de corrientes superficiales predominantes en la región, como por ejemplo la corriente de Panamá (20), la cual parece funcionar como un corredor de distribución y recepción de larvas de crustáceos (6). De esta manera, algunas de las especies logran dispersarse hacia islas como Malpelo en Colombia y Galápagos en Ecuador, a la vez las larvas parecen migrar al norte y llegar a localidades como el golfo de California usando corrientes como la de Costa Rica. Estos mecanismos de dispersión parecen ser los responsables de la gran afinidad que tiene el Pacífico colombiano con las provincias Pacífico nororiental cálidotemplado y Galápagos.

En conclusión los galateoideos en aguas colombianas presentan ámbitos geográficos y batimétricos amplios, presentándose simpatría entre algunas especies. Las de aguas someras presentan mayores restricciones que aquellas de distribución en aguas profundas. Los mecanismos de distribución de las especies corresponden con el ciclo de vida de cada una y las corrientes predominantes en las provincias.

\section{REFERENCIAS}

1. Díaz JM, Acero A. Marine biodiversity in Colombia: Achievements, status of knowledge and challenges. Gayana 2003; 67(2):261-274.

2. Baba K. Deep-sea Chirostylid and Galatheid crustaceans (Decapoda: Anomura) from the Indo-pacific, with a list of species. Galathea Report $2005 ; 20: 1-317$.

3. Baba K, Macpherson E, Poore GCB, Ahyong ST, Bermúdez A, Cabezas P, et al Catalogue of squat lobsters of the world (Crustacea: Decapoda: Anomura - families Chirostylidae, Galatheidae and Kiwaidae). Zootaxa 2008; 1905:1-220.

4. Campos NH, Navas GR, Bermúdez A y Cruz N. Los crustáceos decápodos de la franja superior del talud continental (300 - $500 \mathrm{~m}$ ) del mar Caribe colombiano. Bogotá: Universidad Nacional de Colombia, Facultad de Ciencias Naturales; 2005.
5. Spalding MD, Fox HE, Allen GR, Davison N, Ferdaña ZA, Finlayson $M$ et al Marine Ecoregions of the World: A Bioregionalization of Coastal and Shelf Areas. BioScience 2007; 57(7):573-583.

6. Boschi EE. Species of decapod crustaceans and their distribution in the American marine zoogeographic provinces. Rev Invest Des Pesq 2000; 13:7-136.

7. Navas GR. Taxonomía, distribución y posibles eventos de especiación y dispersión de los crustáceos galatéideos (Decapoda: Anomura) presentes en aguas colombianas. [Tesis Doctoral]. Santa Marta: Universidad Nacional de Colombia; 2011.

8. Melo-Filho GAS. O gênero Munida Leach (Crustacea: Decapoda: Galatheidae) no Atlântico e Mediterrâneo: composicâo e biogeografia. [Tesis Doctoral]. São Paulo: Universidad de São Paulo; 1997. 
9. Pequegnat LH y Pequegnat WE. Deep-sea anomurans of superfamily Galatheoidea with description of three new species. En: Pequegnat WE y Chace FA (eds). Contributions on the Biology of the Gulf of Mexico. College Station: Texas A \& M University; 1970.

10. Hendrickx ME. The temperate species of the genus Munida Leach (Crustacea, Decapoda, Galatheidae) in the East Pacific, with the description of a new species and additional records for tropical-subtropical species. Bull Inst R Sci Nat Belg Biol 2003; 73:115-136.

11. Macpherson E. y M. Segonzac. Species of the genus Munidopsis (Crustacea, Decapoda, Galatheidae) from the deep Atlantic Ocean, including cold-seep and hydrothermal vent areas. Zootaxa 2005; 1095:1-60.

12. Macpherson E. Species of the genus Munidopsis Whiteaves, 1784 from the Indian and Pacific Oceans and reestablishment of the genus Galacantha A. Milne-Edwards, 1880 (Crustacea, Decapoda, Galatheidae). Zootaxa 2007; 1417:1-135.

13. Wilson GD, Hessler RR. Speciation in the deep sea. Annu Rev Ecol Evol Syst 1987; 18:185-207.

14. Palumbi S.R. Populations genetics, demographic connectivity and the design of marine protected areas. Ecological Aplications 2003; 13:146-158.

15. Gore HR. Larval development of Galathea rostrata under laboratory conditions, with a discussion of larval development in the Galatheidae (Crustacea Anomura). Fish Bull 1979; 76:781-806.
16. Konishi K, Saito T. Larvae of the deep-sea squat lobsters, Agononida incerta (Henderson, 1888) and Munida striola Macpherson and Baba, 1993 with notes on larval morphology of the family (Crustacea: Anomura: Galatheidae). Zoological Science 2000; 17:1021-1029.

17. Bermúdez A, Navas GR, Campos NH. Populationsstruktur und genetische Isolierung von drei Springkrebs-Arten. Der Einfluss der ozeanographischen Merkmale und der geographischen Distanz zwischen den Populationen entlang der karibischen Küste von Kolumbien. VDM Verlag, Saarbrücken; 2008.

18. Miloslavich P, Klein E, Díaz JM, Hernández CE, Bigatti G, Campos L, et al Marine Biodiversity in the Atlantic and Pacific Coasts of South America: Knowledge and Gaps. PLoS ONE $2001 ; 6(1)$ :e14631. doi:10.1371/journal. pone.0014631.

19. Cowen RK, Paris CB, Srinivasan A. 2006. Scaling of connectivity in Marine populations. Science 2006; 311:522-527.

20. Lemaitre R, Álvarez-León R. Crustáceos decápodos del Pacífico colombiano: lista de especies y consideraciones zoogeográficas. An Inst Invest Mar Punta Betin 1992; 21:33-76. 